\title{
Aptamer-functionalized AlGaN/GaN High-electron-mobility Transistor for Rapid Diagnosis of Fibrinogen in Human Plasma
}

\author{
Tse-Yu Tai, ${ }_{1}^{1}$ Anirban Sinha, ${ }^{1}$ Indu Sarangadharan, ${ }^{1}$ Anil Kumar Pulikkathodi, ${ }^{1}$ \\ Shin-Li Wang, ${ }^{1}$ Shu-Chu Shiesh, ${ }^{4}$ Gwo-Bin Lee, ${ }^{1,2,3^{*}}$ and Yu-Lin Wang ${ }^{1,2^{* *}}$ \\ ${ }^{1}$ Institute of Nanoengineering and Microsystems, National Tsing Hua University, Hsinchu 300, Taiwan, R.O.C. \\ ${ }^{2}$ Department of Power Mechanical Engineering, National Tsing Hua University, Hsinchu 300, Taiwan, R.O.C. \\ ${ }^{3}$ Institute of Biomedical Engineering, National Tsing Hua University, Hsinchu 300, Taiwan, R.O.C. \\ ${ }^{4}$ Department of Medical Laboratory Science and Biotechnology, \\ National Cheng Kung University, Tainan City 701, Taiwan, R.O.C.
}

(Received March 5, 2018; accepted June 28, 2018)

Keywords: cardiovascular disease, fibrinogen, aptamer, $\mathrm{AlGaN} / \mathrm{GaN}$ HEMT biosensor, in vitro diagnostics

In this research, we have developed a rapid diagnostic assay using a high-electron-mobility transistor (HEMT)-based biosensor to screen for fibrinogen in clinical plasma samples. High sensitivity over a wide detection range ( $0-5 \mathrm{~g} / \mathrm{L})$, good selectivity, low-cost fabrication, portability, and simplicity of use are key features of our biosensor. Early diagnosis of heart disease in low-, medium-, and high-risk patients is possible with an assay turn-around time of 5 min and a plasma sample volume of $5-10 \mu \mathrm{L}$. High sensitivity and low detection limits $(0.5$ $\mathrm{g} / \mathrm{L}$ ) are made possible in environments with high salt concentrations because the HEMT sensor design overcomes the charge screening effect associated with a very small Debye length. This biosensor technology demonstrates the potential for development as a point-of-care or homecare diagnostic device for quick and easy assessment of cardiovascular disease (CVD) risk.

\section{Introduction}

Cardiovascular diseases (CVDs) have been the leading cause of morbidity and mortality worldwide for several years. Every year, 17.5 million people die of CVDs according to World Health Organization. Ischemic heart disease and stroke are the world's biggest killers, accounting for combined 15 million deaths in 2015, which include $80 \%$ of CVD deaths. ${ }^{(1)}$ With increasing age, the risk of suffering from CVDs increases. In addition to age, other factors that affect the risk of CVDs in patients are genetics, diet, work, and lifestyle. Recent research has claimed that about $82 \%$ of patients that suffer from coronary heart disease and stroke are 65 years of age or older. ${ }^{(2)}$ As the mortality rate due to CVD rises even in this age of medical advancement, health care facilities and institutions are now paying more attention to CVD prevention, diagnosis, and treatment. CVDs include hypertension (high blood pressure),

\footnotetext{
*Corresponding author: e-mail: gwobin@pme.nthu.edu.tw

${ }^{* *}$ Corresponding author: e-mail: ylwang@mx.nthu.edu.tw https://doi.org/10.18494/SAM.2018.1938
} 
coronary heart disease, cerebrovascular disease (stroke), peripheral vascular disease, heart failure, rheumatic heart disease, congenital heart disease, and cardiomyopathies. ${ }^{(3)}$

Biomarkers have been used for diagnosis and prognosis since the 1980s. ${ }^{(4)}$ Fibrinogen, which is a glycoprotein responsible for forming fibrin and fibrin-based blood clots, ${ }^{(5)}$ is an important cardiac marker with specific pathophysiology in coronary artery disease and thrombosis. Studies have shown that people who have a fibrinogen concentration above $402 \mathrm{mg} / \mathrm{dl}$ have a $7.7 \%$ mortality rate, while for those with a concentration of less than $402 \mathrm{mg} / \mathrm{dl}$, it was only $3.1 \%$, a twofold difference between high-level mortality and low-level mortality. ${ }^{(5)}$ Plasmatic fibrinogen is analyzed in clinics primarily using the Clauss method, which is a functional assay used to determine fibrinogen concentration from the time taken for a clot to form. ${ }^{(6)}$ Another method is a gravimetric assay that measures the weight of the clot formed. ${ }^{(7)}$ These techniques have long turn-around times and are difficult in terms of sample processing. Hence, these assays are performed only in hospital-based settings. The normal range of plasmatic fibrinogen is $1.5-3$ $\mathrm{g} / \mathrm{L} .^{(8,9)}$ Fibrinogen concentrations $>3.43 \mathrm{~g} / \mathrm{L}$ are relevant to CVDs. The traditional assays suffer from inconsistencies in determining fibrinogen concentration. ${ }^{(10)}$ Thus, a rapid and robust technique for the determination of fibrinogen is important to fully utilize the diagnostic value of this cardiac marker in CVD risk assessment and prevention.

In this paper, we report the development of a miniaturized electronic sensor for the rapid screening of fibrinogen, based on the molecular recognition of plasmatic fibrinogen by a singlestranded DNA probe (aptamer). Field-effect transistors (FETs) are attractive for biosensing applications due to their high signal-to-noise ratio, ease of integration, and low cost. Compound semiconductor-based FETs, such as AlGaN/GaN high-electron-mobility transistors (HEMTs), have been used in biomedical applications owing to their superior electrical and chemical properties. ${ }^{(11,12)}$ They are chemically inert and hence resistant to the harsh environment of physiological fluids. On the other hand, conventional Si-based FET sensors suffer from degradation or shift in electrical response due to the hydrolysis of the oxide layer in aqueous solutions. Moreover, GaN HEMTs can also be batch-fabricated using standard semiconductor processing and offer superior thermal resistance and high sensitivity. Traditional FET biosensors, however, suffer from severe charge screening effects in physiological fluids due to high ionic strength. ${ }^{(13-15)}$ Previously, we developed a FET biosensor-based method to directly detect protein biomarkers in human serum. ${ }^{(16-21)}$ In this study, we have developed an aptamerfunctionalized GaN HEMT biosensor to directly detect fibrinogen in untreated human plasma samples. The charge screening effect is eliminated using a high-field gating mechanism that is uniquely employed in this FET sensor methodology. The sensor demonstrates high sensitivity over a wide dynamic detection range. This technology can be developed as an in vitro diagnostic device for home-care or point-of-care testing.

\section{Experimental Methods}

\subsection{AlGaN/GaN HEMT fabrication}

The HEMT structure includes a 3- $\mu \mathrm{m}$-thick undoped GaN layer and a 150 - $\mu \mathrm{m}$-thick $\mathrm{Al}_{0.25} \mathrm{Ga}_{0.75} \mathrm{~N}$ layer. A two-dimensional electron gas (2DEG) forms at the interface between 
the $\mathrm{AlGaN}$ and $\mathrm{GaN}$ layers. The $\mathrm{AlGaN} / \mathrm{GaN}$ is deposited by metal organic chemical vapor deposition (MOCVD). All spatial structures are defined by photolithography. Mesa isolations are formed on the epi wafer to create a channel (shown in Fig. 1). This is done using inductively coupled plasma (ICP) in $\mathrm{Cl}_{2} / \mathrm{BCl}_{3}$ gases at an ICP power of $300 \mathrm{~W}$ and an RF bias of $120 \mathrm{~W}$ at $2 \mathrm{MHz}$. Once a channel is formed, the drain-source ohmic metal contacts are deposited as layers of $200 \AA$ of Ti, $400 \AA$ of $\mathrm{Al}, 800 \AA$ of Ni, and $1000 \AA$ of Au using an electron beam evaporation system (E-beam). The layers undergo rapid thermal annealing (RTA) twice. The first annealing is performed at $200{ }^{\circ} \mathrm{C}$ for $25 \mathrm{~s}$, and the second annealing, at $850{ }^{\circ} \mathrm{C}$ for $40 \mathrm{~s}$ in an inert environment of $\mathrm{N}_{2}$. Following this, the metal interconnects and gate electrode separate from the channel that consists of $200 \AA$ of Ti and $1000 \AA$ of Au. The entire device is passivated using a photoresist, and the gate electrode and FET channel are selectively opened using photolithography. The schematics and top view are shown in Fig. 2. The fabricated HEMT chip can be embedded in a polymer substrate with in-plane interconnects, which can then be easily integrated with a portable biosensor system. The FET packaging process is described in detail in our previous works. ${ }^{(17,22)}$

\subsection{Aptamer selection}

The aptamer was selected by the systematic evolution of ligands by exponential enrichment (SELEX). ${ }^{(23)}$ Initially, magnetic beads surface-coated with fibrinogen were used. Then, the beads and single-stranded DNA (ssDNA) library (concentration $=1 \mathrm{M}$, Medclub Scientific Co., Ltd., Taiwan) were mixed such that ssDNA with high affinity towards fibrinogen attached to the surface of the beads. Then, a magnetic field was applied to collect ssDNA-bead complexes. The unbound ssDNA was removed by washing. The captured ssDNA with high affinity towards fibrinogen was heated to release ssDNA and amplified by subsequent polymerase chain reaction (PCR). After several repeated cycles, a specific aptamer with high affinity towards fibrinogen was obtained. ${ }^{(23)}$ The dissociation constant of the aptamer was measured as $100 \mathrm{nM}$.

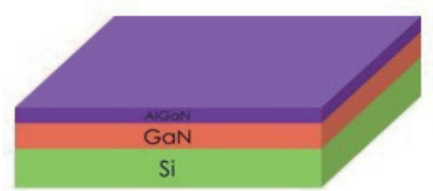

(a)

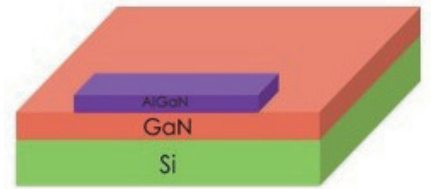

(b)

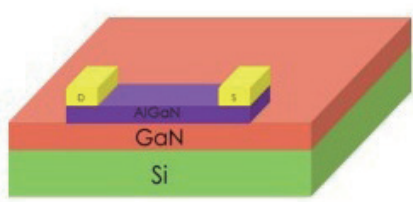

(c)

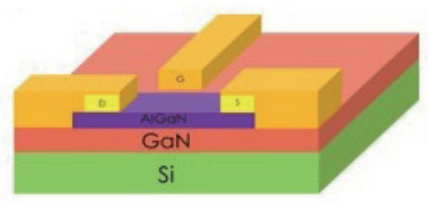

(d)

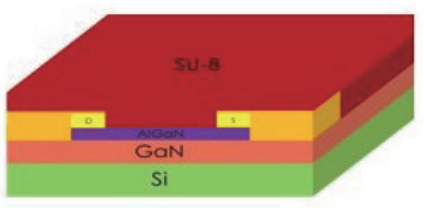

(e)

Fig. 1. (Color online) Schematic of the HEMT biosensor fabrication process: (a) structure of the epi wafer, (b) defining the active area by ICP etching, (c) ohmic contact metal (Ti/Al/Ni/Au) deposited with E-beam and annealing, (d) final metal ( $\mathrm{Ti} / \mathrm{Au})$ deposition with E-beam, and (e) passivation of the photoresist on the device. 


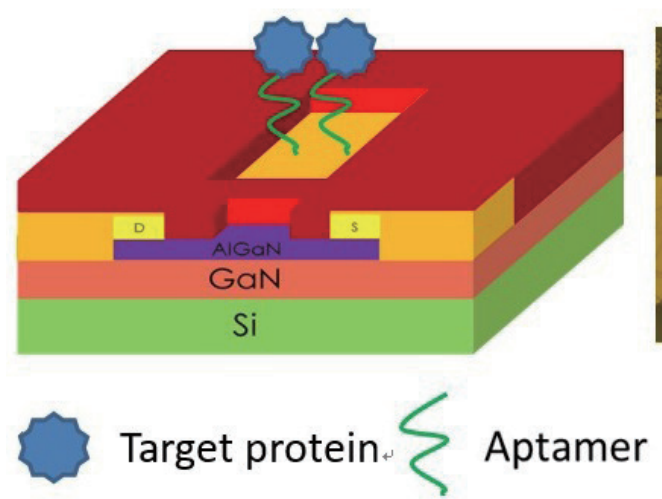

(a)

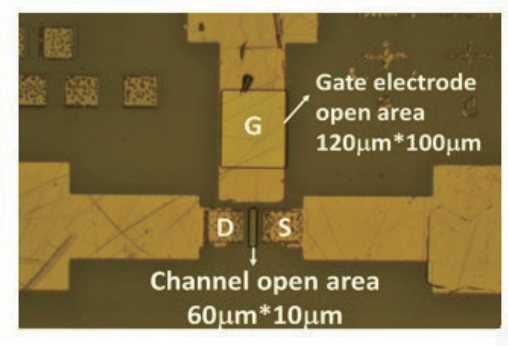

(b)

Fig. 2. (Color online) (a) Schematic diagram of GaN HEMT biosensor and (b) top view of the HEMT chip describing the sensing region.

\subsection{Aptamer immobilization}

The sensor chip was initially cleaned using a UV-ozone system and washed in dilute acid and deionized water. The aptamer used for immobilization was fibrinogen-specific ssDNA with dithiol modification on the 5' side. A $5 \mu \mathrm{M}$ solution of aptamer was prepared in Trisethylenediaminetetraacetic acid (EDTA) buffer (TE buffer). Aptamer solution $(20 \mu \mathrm{L})$ was mixed with $2 \mu \mathrm{L}$ of $1 \mathrm{mM}$ tris(2-carboxyethyl) phosphine (TCEP). TCEP is a reducing agent for the dithiol on the aptamer (ssDNA) 5' side. The aptamer solution was heated to $95{ }^{\circ} \mathrm{C}$ and later dropped on the sensor surface and incubated at room temperature for $24 \mathrm{~h}$. The aptamer immobilization process is schematically depicted in Fig. 3(a). The surface functionalization can be electrically verified by measuring the signal before and after aptamer immobilization. The results are depicted in Fig. 3(b).

\subsection{Purified protein samples in $1 \times$ phosphate-buffered saline (PBS)}

Fibrinogen protein was purchased from Abcam (catalog \#ab84410, United Kingdom). It was diluted to physiological concentrations in $1 \times$ PBS $(137 \mathrm{mM} \mathrm{NaCl}, 2.7 \mathrm{mM} \mathrm{KCl}, 10 \mathrm{mM}$ $\mathrm{Na}_{2} \mathrm{HPO}_{4}, 2 \mathrm{mM} \mathrm{KH} \mathrm{PO}_{4}, \mathrm{pH}$ adjusted to 7.4 with $\mathrm{NaOH}$ ) and contained $4 \%$ bovine serum albumin (BSA) to simulate human blood plasma.

\subsection{Clinical human blood plasma samples}

Clinical samples of human plasma containing fibrinogen were obtained as per approval by the institutional review board (IRB) of National Cheng-Kung University Hospital (NCKUH) (IRB. No. B-ER-104-116) and under the supervision of National Tsing Hua University IRB (10405HE014). The concentration range of fibrinogen was $1.72-6.94 \mathrm{~g} / \mathrm{L}$. The clinical plasma samples were directly used for electrical detection without any preprocessing. 


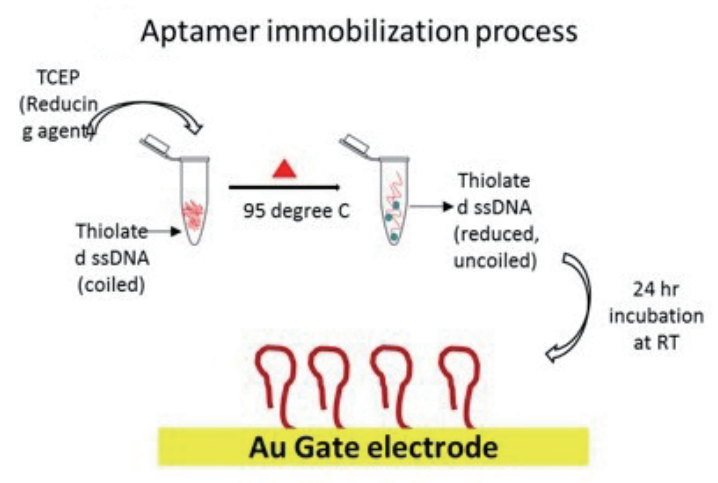

(a)

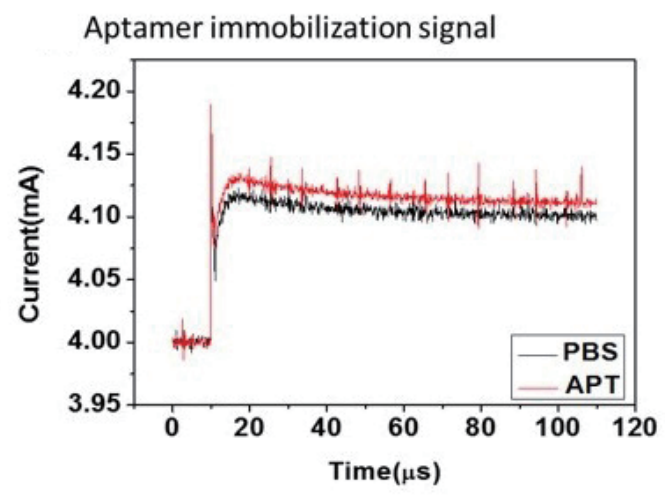

(b)

Fig. 3. (Color online) (a) Schematic diagram of the immobilization process and (b) electrical characteristics before and after aptamer immobilization. The measurement setup and details of electrical characterization are shown in Sect. 2.6.

\subsection{Measurement using the sensor}

The electrical signals from the sensor were measured using an Agilent B1530/B1500A semiconductor parameter analyzer. The bias condition consisted of a steady DC bias of $2 \mathrm{~V}$ on the drain, here referred to as $V_{d}$. A short-duration $(100 \mu \mathrm{s})$ pulse of $1.5 \mathrm{~V}$ was applied to the gate electrode; $V_{g}$. When the pulsed $V_{g}$ was applied, the electrical signal passed through the gate electrode to the channel via the test solution and modulated the channel conductivity.

\subsection{Protein elution}

After testing the sensor with a fibrinogen-containing solution (buffer and plasma), the sensor was repeatedly washed in deionized (DI) water heated to $40{ }^{\circ} \mathrm{C}$ to elute the bound and unbound proteins from the sensor surface. The device was later rinsed in $1 \times$ PBS. The sensor baseline was verified electronically to confirm sensor regeneration.

\section{Results and Discussion}

As FET biosensors are always used in aqueous solutions, charge screening effects occur at the solid/liquid interface preventing the biomolecule-induced potential changes from modulating channel conductivity. ${ }^{(16)}$ The thickness of the electrical double layer (EDL) at the interface can be described by the parameter called Debye length, defined in the Debye-Huckel equation as

$$
k^{-1}=\sqrt{\frac{\varepsilon_{r} \varepsilon_{0} k_{B} T}{2 N_{A} e^{2} I}} .
$$

Here, $k^{-1}$ is the Debye length, $\varepsilon_{0}$ is the dielectric constant, $\varepsilon_{0}$ is the free space permittivity, $k_{B}$ 
is the Bolzmann constant, $T$ is the temperature, $N_{A}$ is Avogadro's number, $e$ is the elementary charge, and $I$ is the ionic strength of electrolyte solution. The Debye length decreases as the ionic strength of the electrolyte solution increases; this phenomenon is called the charge screening effect. ${ }^{(13-15)}$ Typically, this is eliminated by diluting the salt concentration of the test solution 1000-10000 times so that the Debye length increases and eliminates charge screening. ${ }^{(24,25)}$ However, such sample processing techniques are tedious and adversely affect the binding kinetics and activity of biomolecular reaction. Previously, we employed a different strategy to eliminate the effects of charge screening without the need for sample processing. ${ }^{(16)}$ A high electric field was applied across the test solution via a separate gate electrode that does not contact the FET channel. This electric field instantly modulated the EDLs at the gate electrode and HEMT dielectric $(\mathrm{AlGaN})$ interfaces within the solution, creating a solution capacitance. This capacitance modulates the potential drop across the HEMT dielectric and hence the channel conductivity. If $V_{g}$ is the voltage applied to the gate electrode, $V_{g}$ drops across the solution and HEMT dielectric before grounding, as described in Eq. (2):

$$
V_{g}=\Delta V_{s}+\Delta V_{d}
$$

where $V_{s}$ and $V_{d}$ are the potential drops across the test solution and FET dielectric, respectively.

Considering the impedance across the sensor structure, the solution capacitance generated by EDL formation and the HEMT dielectric capacitance are assumed to be a parallel combination of capacitors. Thus, we can represent $V_{d}$ in Eq. (2) as

$$
\Delta V_{d}=\frac{\frac{1}{j \omega C_{d}}}{\frac{1}{j \omega C_{d}}+\frac{1}{j \omega C_{s}}} \times V_{g}=\frac{C_{s}}{C_{d}+C_{s}} \times V_{g}
$$

where $C_{d}, C_{S}$, and $\omega$ are the dielectric capacitance, solution capacitance, and angular frequency, respectively.

This means that, when $C_{s}$ changes, the potential drop across the dielectric, $V_{d}$, changes, resulting in changes in HEMT drain current. As seen in Fig. 3, when the sensor surface is functionalized and a high field is applied across the test solution, the EDL in the gate electrode region is modified, which subsequently changes the EDL charge distribution in the dielectric region, thereby changing $C_{s}$. As a result, $V_{d}$ changes and the drain current is modulated accordingly. This is depicted in the graph in Fig. 3(b), which shows the changes in sensor signal upon surface functionalization. Similarly, when a target molecule binds with the receptor, the aptamer in this case, the sensor signal changes as a result of changes in $C_{s}$ and $V_{d}$.

\subsection{Detection of purified fibrinogen in $1 \times$ PBS with $4 \%$ BSA}

Different concentrations of fibrinogen prepared in buffer solution were measured after aptamer immobilization was confirmed. The protein solutions were prepared in $1 \times$ PBS 
with $4 \%$ BSA. The sensor measurement results for different fibrinogen concentrations are shown in Fig. 4. The drain current signal was measured at a sampling rate of $10 \mathrm{~ns}$ during the period of gate pulsed voltage application. The graph of drain current versus time for different fibrinogen concentrations is shown in Fig. 4(a). To distinguish the signal from different protein concentrations, we used another sensor index called total charge. It is defined as

$$
Q=\int I_{d} d t
$$

where $Q$ is the calculated total charge and $I_{d}$ is the drain current. The $Q$ value does not represent the physical surface charges that accumulate on the sensor but is rather a sensor index used for calibration. Upon integrating the drain current, we can obtain good separation between different fibrinogen concentrations, as seen in Fig. 4(b). Using this graph, we can derive a sensor calibration curve, as seen in Fig. 4(c), which shows the total charge versus fibrinogen concentration. The drain current decreased as the fibrinogen concentration increased. The sensor exhibited a high sensitivity with a linear response over a wide dynamic range (0-4.5 g/L).

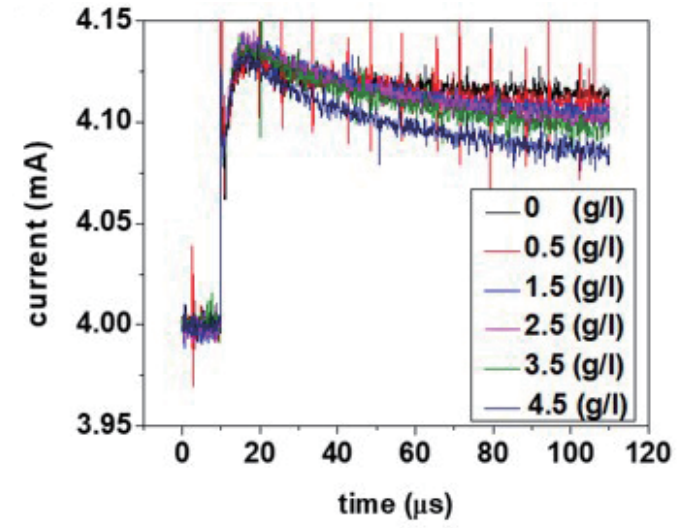

(a)

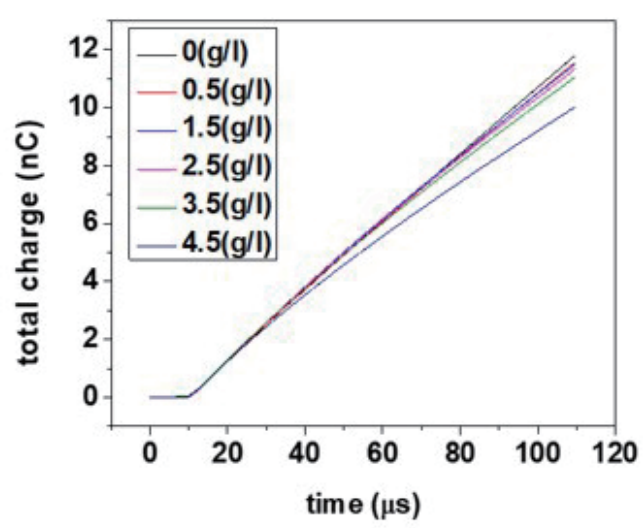

(b)

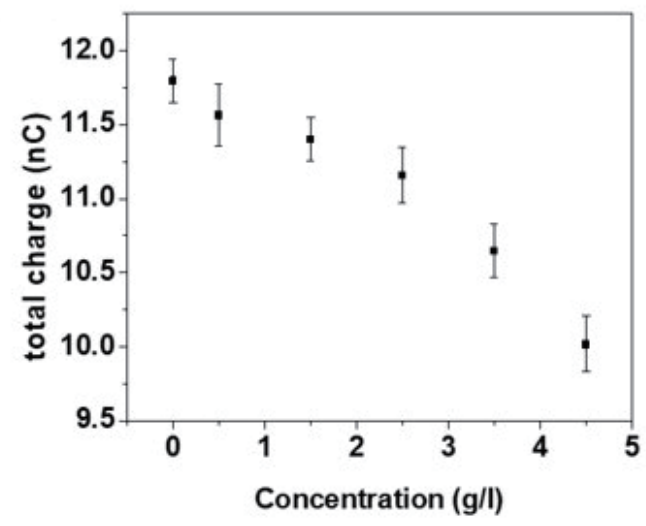

(c)

Fig. 4. (Color online) Fibrinogen detection in $1 \times$ PBS with 4\% BSA: (a) graph of drain current versus time, (b) graph of total charge versus time, and (c) sensor calibration curve. 


\subsection{Detection of plasmatic fibrinogen}

After validating the assay using purified proteins prepared in $1 \times$ PBS with $4 \%$ BSA, we carried out experiments with human plasma. Previously, we evaluated the electrical response of human serum to a sensor without receptors. ${ }^{(16,26-28)}$ The background biomolecules present in human serum did not cause a nonspecific signal. We used a sample incubation period of 5 min, during which the specific aptamer-protein binding dominated other nonspecific binding processes. The strong electrostatic interaction of the aptamer-protein moiety created a local charge redistribution at the EDL, which resulted in changes in $C_{S}$. However, nonspecific binding did not create such strong electrostatic interactions and hence did not significantly alter the sensor response. This behavior contributed to good sensor selectivity, which is an important characteristic for clinical diagnostics. To evaluate the sensitivity and selectivity of our sensor, we obtained clinical samples of human plasma containing different fibrinogen concentrations: $1.72,2.73,3.54,4.21$, and $5.21 \mathrm{~g} / \mathrm{L}$. The plasma samples were untreated and used for electrical measurements without any further processing. The electrical characteristics of the tests using plasmatic fibrinogen are shown in Fig. 5. The graph of drain current versus time and the

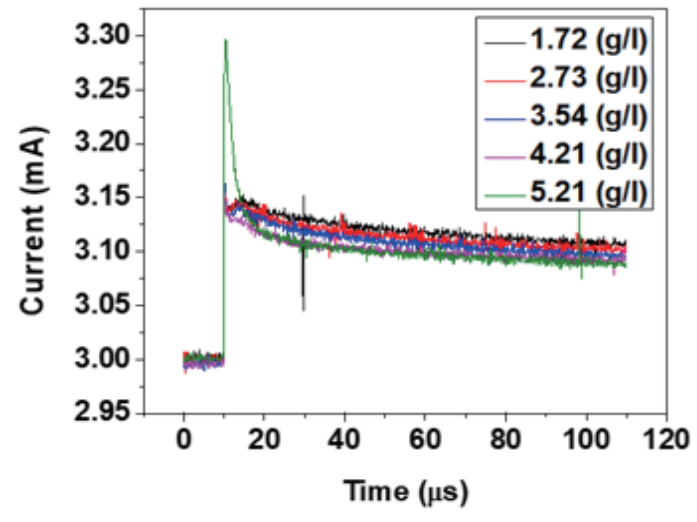

(a)

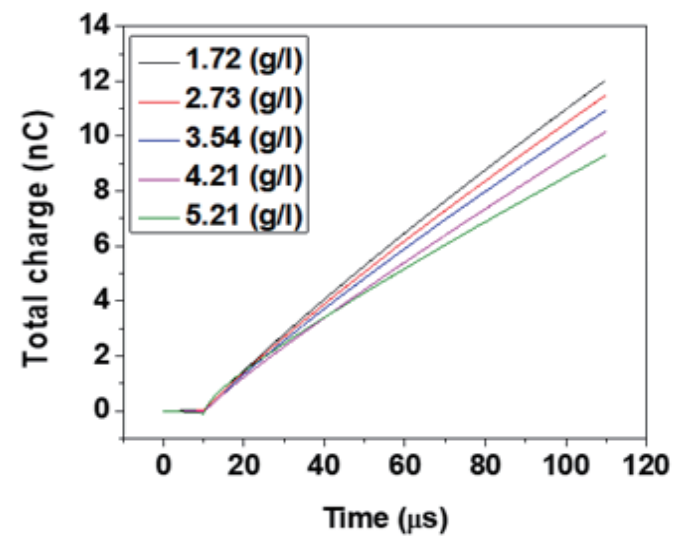

(b)

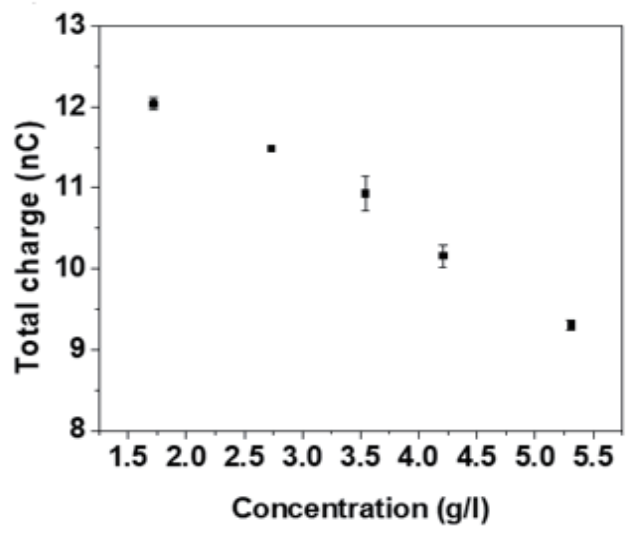

(c)

Fig. 5. (Color online) Detection of fibrinogen in clinical plasma samples: (a) graph of drain current versus time, (b) graph of total charge versus time, and (c) sensor calibration curve. 
corresponding graph of total charge versus time are shown in Figs. 5(a) and 5(b), respectively. Figure 5(c) shows the sensor calibration curve. Similar to the sensing characteristics in buffer (as seen in Fig. 4), we observed good sensitivity over a wide dynamic detection range. The clinical plasma samples also contained a large variety of background proteins and other biomolecules, but the sensor exhibited good dependence on fibrinogen concentration. The sensor response to nonspecific entities in different patient samples has also been evaluated and demonstrated in our previous works. ${ }^{(16,26)}$ The plasma samples were obtained from different patients at different times, but the sensor calibration curve displays good sensing characteristics, which means that nonspecific binding did not adversely affect the sensor response. This is mainly due to the short sample incubation period of $5 \mathrm{~min}$ during which nonspecific binding is relatively much lower than specific aptamer-protein binding, owing to its much lower reaction rate.

\subsection{Sensor regeneration}

The sensor could be regenerated by eluting the bound target proteins from the sensor surface. After each sample (in buffer and clinical plasma) was measured, the sensor was washed in DI water heated to $40{ }^{\circ} \mathrm{C}$. In DI water, because there are many fewer ions, the electrostatic interaction of aptamer and protein is disrupted, and protein can be effectively washed away from a sensor. After washing, the device was rinsed in $1 \times$ PBS to restore the native structure of the aptamer. Electrical measurements uisng the sensor, which are shown in Fig. 6, demonstrated that the electrical baseline corresponding to the initial aptamer level was restored after elution. Between sample measurements, the sensor surface was regenerated by eluting the bound fibrinogen and other background proteins. In this way, during every test, we could control the electrical response from the sample background by keeping the nonspecific binding to a minimum. In both cases of fibrinogen in buffer and clinical plasma samples, the sensor surface could be cleaned effectively [Figs. 6(a) and 6(b)] using the protein elution-washing procedure.

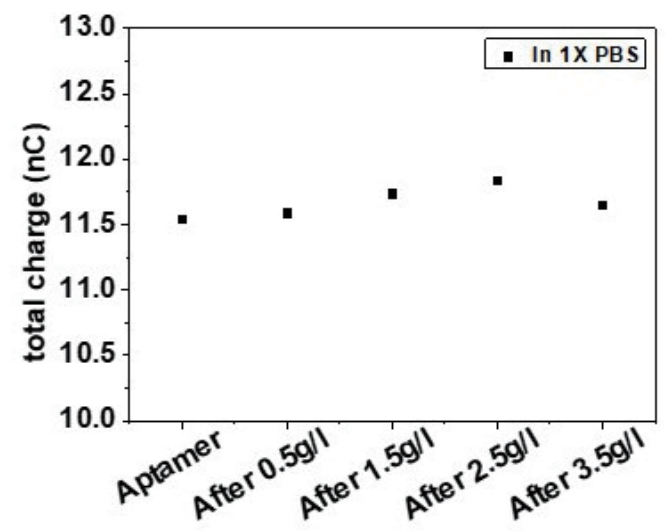

(a)

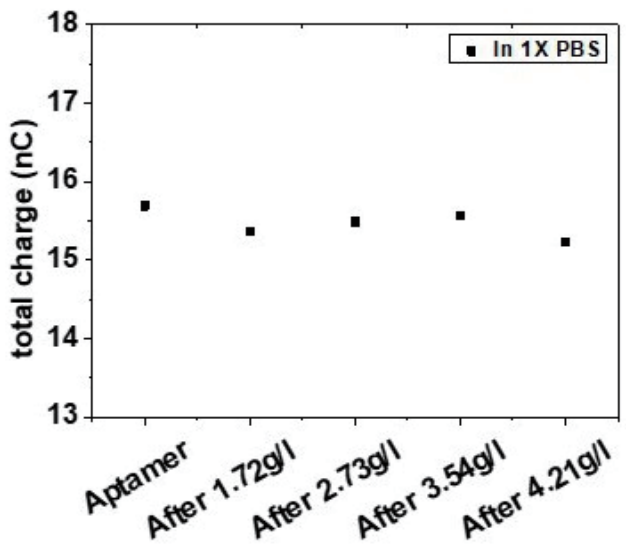

(b)

Fig. 6. Electrical baseline after protein sample measurement in (a) $1 \times$ PBS with $4 \%$ BSA and (b) clinical sample. 


\section{Conclusions}

In this work, we utilized an aptamer-functionalized $\mathrm{AlGaN} / \mathrm{GaN}$ HEMT biosensor to detect plasmatic fibrinogen. The unique sensor design provides high electric field modulation, which eliminates the charge screening effect in high salt environments. This facilitates direct protein detection, even in clinical human plasma samples, without extensive sample pretreatment. Because a nucleotide-based receptor is employed as opposed to a conventional protein-based receptor, the biosensor has a higher stability and a longer shelf life, which are critical characteristics for an in vitro diagnostic device. We demonstrated the sensing and selectivity characteristics of a GaN HEMT biosensor using purified protein samples in $1 \times \mathrm{PBS}$ with 4\% BSA and in clinical samples. The HEMT biosensor can be used in personal health care products as it is inexpensive, easy to integrate into a portable system, and can be used by anyone without prior training or knowledge. ${ }^{(29)}$ In the future, this technology can pave the way for rapid diagnostics of a panel of CVD biomarkers for better disease risk assessment and prevention.

\section{Acknowledgements}

This work was supported by the Ministry of Science \& Technology (MOST 106-2221-E007-002-) and National Tsing Hua University in cooperation with Mackay Memorial Hospital in Hsinchu (106J00X9JA). We also express our thanks to the Center for Nanotechnology, Materials Science, and Microsystems (CNMM) at National Tsing Hua University, and to Epi Star Corporation for their continued support.

\section{References}

1 M. Naghavi, H. Wang, R. Lozano, A. Davis, X. Liang, M. Zhou, S. E. Vollset, and M. A. Bohensky: The Lancet 385 (2015) 10.

2 V. L. Feigin, M. H. Forouzanfar, R. Krishnamurthi, G. A. Mensah, M. Connor, D. A. Bennett, A. E. Moran, R. L Sacco, L. Anderson, T. Truelsen, M. O’Donnell, N. Venketasubramanian, S. Barker-Collo, C. M. Lawes, W. Wang, Y. Shinohara, E. Witt, M. Naghavi, and C. Murray: The Lancet 383 (2014) 245.

3 J. A. Finegold, P. Asaria, and D. P. Francis: Int. J. Cardiol. 168 (2013) 934.

4 J. Aronson: Br. J. Clin. Pharmacol. 59 (2005) 491.

5 T. J. Wang, P. Gona, M. G. Larson, G. H. Tofler, D. Levy, C. N. Cheh, P. F. Jacques, N. Rifai, J. Selhub, S. J. Robins, E. J. Benjamin, R. B. D’Agostino, and R. S. Vasan: New Engl. J. Med. 355 (2006) 2631.

6 G. Palareti and M. Maccaferri: Res. Clin. Lab. 20 (1990) 167.

7 I. J. Mackie, S. Kitchen, S. J. Machin, and G. D. O. Lowe: Br. J. Haematol. 121 (2003) 396.

8 R. Wang, A. L. Khosh, S. Choi, and J. Chae: Biosens. Bioelectron. 28 (2011) 304.

9 G. Ndrepepa, S. Braun, L. King, M. Fusaro, D. Keta, S. Cassese, T. Tada, A. Schömig, and A. Kastrati: Am. J. Cardiol. 111 (2013) 804.

10 P. W. Koppert, E. Hoegee-de Nobel, and W. Nieuwenhuizen: Thromb. Haemost. 59 (1988) 310.

11 Y. L. Wang, B. H. Chu, K. H. Chen, C. Y. Chang, T. P. Lele, Y. Tseng, S. J. Pearton, J. Ramage, D. Hooten, A. Dabiran, P. P. Chow, and F. Ren: Appl. Phys. Lett. 93 (2008) 262101.

12 B. H. Chu, B. S. Kang, C. Y. Chang, F. Ren, A. Goh, A. Sciullo, W. Wu, J. Lin, B. P. Gila, S. J. Pearton, J. W. Johnson, E. L. Piner, and K. J. Linthicum: IEEE Sens. J. 10 (2010) 64.

13 K. I. Chen, B. R. Li, and Y. T. Chen: Nano Today 6 (2011) 131.

14 C. P. Hsu, Y. F. Huang, and Y. L. Wang: ECS J. Solid State Sci. Technol. 5 (2016) Q149.

15 E. Stern, R. Wagner, F. J. Sigworth, R. Breaker, T. M. Fahmy, and M. A. Reed: Nano Lett. 7 (2007) 3405.

16 C. H. Chu, I. Sarangadharan, A. Regmi, Y. W. Chen, C. P. Hsu, W. H. Chang, G. Y. Lee, J. I. Chyi, C. C. Chen, S. C. Shiesh, G. B. Lee, and Y. L. Wang: Sci. Rep. 7 (2017) 5256. 
17 P. C. Chen, Y. W. Chen, I. Sarangadharan, C. P. Hsu, C. C. Chen, S. C. Shiesh, G. B. Lee, and Y. L. Wang: ECS J. Solid State Sci. Technol. 6 (2017) Q71.

18 A. K. Pulikkathodi, I. Sarangadharan, Y. H. Chen, G. Y. Lee, J. I. Chyi, G. B. Lee, and Y. L. Wang: ECS J. Solid State Sci. Technol. 7 (2018) Q3001.

19 A. Regmi, I. Sarangadharan, Y. W. Chen, C. P. Hsu, G. Y. Lee, J. I. Chyi, S. C. Shiesh, G. B. Lee, and Y. L. Wang: Appl. Phys. Lett. 111 (2017) 082106.

20 Y. W. Chen, T. Y. Dai, C. P. Hsu, I. Sarangadharan, A. K. Pulikkathodi, S. H. Wang, R. Sukesan, H. Y. Lin, K. W. Kao, C. L. Hsu, C. C. Chen, and Y. L. Wang: Sens. Actuators, B 262 (2018) 365.

21 J. Yang, P. Carey, F. Ren, Y. L. Wang, M. L. Good, S. Jang, M. A. Mastro, and S. J. Pearton: Appl. Phys. Lett. 111 (2017) 202104.

22 C. P. Hsu, P. C. Chen, A. K. Pulikkathodi, C. C. Chen, and Y. L. Wang: ECS J. Solid State Sci. Technol. 6 (2017) Q63.

23 W. C. Kao, Y. W. Chen, C. H. Chu, W. H. Chang, S. C. Shiesh, Y. L. Wang, and G. B. Lee: Biomicrofluidics 11 (2017) 044105.

24 E. Stern, A. Vacic, N. K. Rajan, J. M. Criscione, J. Park, B. R. Ilic, D. J. Mooney, M. A. Reed, and T. M. Fahmy: Nat. Nano 5 (2010) 138.

25 G. Zheng, F. Patolsky, Y. Cui, W. U. Wang, and C. M. Lieber: Nat. Biotechnol. 23 (2005) 1294.

26 I. Sarangadharan, A. Regmi, Y. W. Chen, C. P. Hsu, P. C. Chen, W. H. Chang, G. Y. Lee, J. I. Chyi, S. C. Shiesh, G. B. Lee, and Y. L. Wang: Biosens. Bioelectron. 100 (2018) 282.

27 I. Sarangadharan, S. L. Wang, T. Y. Tai, A. K. Pulikkathodi, C. P. Hsu, H. H. K. Chiang, L. Y. M. Liu, and Y. L. Wang: Biosens. Bioelectron. 107 (2018) 259.

28 I. Sarangadharan, S. L. Wang, R. Sukesan, P. C. Chen, T. Y. Tai, A. K. Pulikkathodi, C. P. Hsu, H. H. K. Chiang, L. Y. M. Liu, and Y. L. Wang: Anal. Chem. 90 (2018) 2867.

29 C. M. Kuan, S. T. Lin, T. H. Yen, Y. L. Wang, and C. M. Cheng: Biomicrofluidics 10 (2016) 034118. 